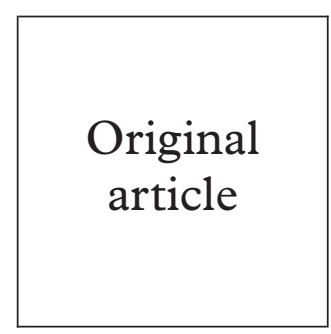

\title{
Poor reduction of HIV-1 RNA titres in nucleoside reverse transcriptase inhibitor experienced patients treated with indinavir combination therapy
}

\author{
J K Ball, T Rowe, R Curran, W L Irving, G M Beards, G Sontag, M Youle, G Moyle, \\ D Pillay
}

Objectives: The long term effectiveness of combination therapy at reducing viral loads in seminal fluid and blood plasma obtained from HIV-1 infected men who had undergone previous antiretroviral therapy was assessed.

Methods: Samples of semen and blood were obtained from a cohort of 12 nucleoside reverse transcriptase inhibitor experienced men before and during 25-68 weeks of combination therapy, which included the protease inhibitor indinavir. HIV-1 RNA titres present in the cell free blood and seminal plasma samples were determined using the nucleic acid sequence based amplification (NASBA)/Nuclisens assay system.

Results: Viral RNA was detected in 9/12 and 7/12 baseline blood plasma and seminal plasma samples, with median viral titres of $10^{4.81}$ and $10^{4.56}$ per $\mathrm{ml}$, respectively. By the end of the study period the detection rates of HIV RNA in the blood and seminal plasma samples were 5/12 and $2 / 12$, respectively, with the median viral titres below the assay cut off level for both sample types. Of the nine patients who had detectable viral RNA in the baseline sample, only three cleared virus from both compartments by the end of the study.

Conclusions: These data show that stable reduction of blood and seminal fluid viral titres is not achievable in a significant proportion of nucleoside reverse transcriptase inhibitor experienced men. (Sex Transm Inf 1999;75:337-339)

Keywords: HIV-1; semen; combination therapy; viral titres; NASBA/Nuclisens

Division of

Microbiology and

Infectious Diseases,

Department of Clinical

Laboratory Sciences,

University of

Nottingham, Queen's

Medical Centre,

Nottingham NG7 2UH

J K Ball

T Rowe

R Curran

W L Irving

PHLS Antiviral

Susceptibility

Reference Unit,

Birmingham Public

Health Laboratory,

Birmingham

Heartlands Hospital,

Birmingham B9 5SS

G M Beards

D Pillay

Kobler Centre, Chelsea and Westminster

Hospital, London

SW10 9TH

G Sontag

G Moyle

\section{Garrett Anderson}

Ward, Royal Free

Hospital, London

NW3 2QG

$M$ Youle

Correspondence to:

Dr J K Ball.

Accepted for publication

18 June 1999

\section{Introduction}

Globally, sexual transmission accounts for the vast majority of new infections of human immunodeficiency virus type 1 (HIV-1). Unfortunately, correlates of transmission of HIV-1 are still poorly understood, but a variety of factors such as the composition and size of the virus reservoir in contaminating body fluids are thought to be important. ${ }^{12}$

Antiretroviral drug combinations, which include recently developed protease inhibitors, are highly effective at reducing viral loads within blood plasma. However, there is increasing evidence that in some individuals distinct populations of HIV can exist in semen compared with blood. ${ }^{13}$ Therefore, if antiretroviral therapy is to have the additional benefit of reducing the risk of sexual transmission by lowering genital tract viral titres, it must demonstrate a potent effect against these distinct viral populations. It is also essential that antiviral therapy is effective against all reservoirs of replicating HIV-1, as drug resistant viruses will evolve during continued replication within sanctuary sites.

Previous studies have shown that potent reduction of genital tract HIV titres frequently occurs following initiation of antiretroviral therapy, particularly combination therapy. ${ }^{45}$ However, the efficacy of combination therapy at reducing genital tract HIV, particularly long term, in nucleoside reverse transcriptase inhibitor (NRTI) experienced individuals is not clear. To address this, we have studied the long term (up to 68 weeks) effect of commencing an indinavir containing combination regimen, in NRTI experienced individuals, on seminal fluid and blood plasma viral RNA titres using the sensitive and internally controlled nucleic acid sequence based amplification (NASBA)/ Nuclisens assay system.

\section{Methods}

SAMPLES

Sequential paired samples of peripheral blood and semen were obtained from HIV-1 infected men before and during combination therapy including indinavir. All the patients had received at least two different NRTIs, either sequentially or in combination, before the study. Samples were collected on the therapy initiation date (baseline) and at various time intervals thereafter, for up to 68 weeks. Cell free plasma was obtained by centrifugation at $1300 \mathrm{~g}$ and plasma samples were stored at $-70^{\circ} \mathrm{C}$ before HIV-1 RNA quantification. All samples were processed within 4 hours of collection.

DETERMINATION OF BLOOD CD4 CELL COUNTS AND SEMINAL FLUID AND BLOOD PLASMA VIRAL RNA TITRES

Blood CD4 cell counts were determined as part of routine patient management. Quantification of viral RNA titres in $50 \mu \mathrm{l}$ samples of seminal fluid and blood plasma was carried out 
using the commercially available NASBA/ Nuclisens assay system. ${ }^{6}$ This volume was found to be the optimal amount of seminal fluid to give maximum sensitivity when using the $900 \mu \mathrm{l}$ lysis volume. Higher inputs of seminal fluid were often associated with either assay failure or unacceptable $Q_{c}$ calibrator values (results not shown). The assay was carried out according to the manufacturer's instructions and the assay cut off was equivalent to 800 $\left(10^{2.90}\right)$ copies per $\mathrm{ml}$ of blood or seminal plasma.

STATISTICAL ANALYSES

All statistical analyses were carried out using non-parametric tests available in the Cstat or Prism for Windows software packages (Cherwell Scientific, Oxford). The Wilcoxon's rank sum test, Spearman's rank correlation coefficient test, Mann-Whitney U test, and Fisher's exact test were used where appropriate. Viral titres below the assay cut off were assigned values of 799 copies per $\mathrm{ml}$.

\section{Results}

Of the 12 patients who provided baseline paired semen and blood samples HIV-1 RNA was detectable in $9 / 12(75 \%)$ and $7 / 12(58 \%)$ pretreatment blood plasma and seminal plasma samples, respectively. The detection rates within the two compartments were not significantly different (Fisher's exact test). By the end of the trial detection rates had decreased to $5 / 12(42 \%)$ and $2 / 12(17 \%)$ in blood and semen samples, respectively. The reduction in detection frequency of HIV-1 RNA in seminal plasma was greater than blood plasma, but neither was significant (Fisher's exact test, $\mathrm{p}=0.0894$ and 0.2138 , respectively).

The effect of treatment upon absolute HIV-1 RNA titres and blood CD4 cell counts is presented in Figure 1. The median RNA titres in blood plasma and seminal fluid before indinavir combination therapy were $10^{4.81}$ (range $\left.<10^{2.90}-10^{5.99}\right)$ and $10^{4.56}\left(<10^{2.90}-10^{5.68}\right)$ copies per $\mathrm{ml}$, respectively, while median blood CD4 cell counts were $11(0-288)$ cells $\times 10^{6} / 1$ of blood. Viral RNA titres were significantly lower in semen compared with the corresponding blood plasma samples ( $p=0.0105$, Wilcoxon's signed rank test). By the end of the trial the median HIV RNA titres in blood and semen were $<10^{2.90}\left(<10^{2.90}-10^{6.30}\right.$ and $<10^{2.90}-10^{4.08}$, respectively), while the median blood CD4 cell count had increased to $128(6-554)$ cells $\times 10^{6} / 1$ of blood (Fig 1). The differences between the pre- and post-treatment median blood CD4 cell counts and seminal plasma HIV RNA titres were both statistically significant (Mann-Whitney $U$ test $p$ values of 0.0012 and 0.0156 , respectively). By contrast, the difference in median values for the pre- and posttreatment blood plasma samples was not significant $(p=0.1347)$.

\section{Discussion}

Although the sexual transmission of HIV-1 might be influenced by a number of host and viral factors, epidemiological evidence ${ }^{2}$ and inference from studies of sexual transmission of
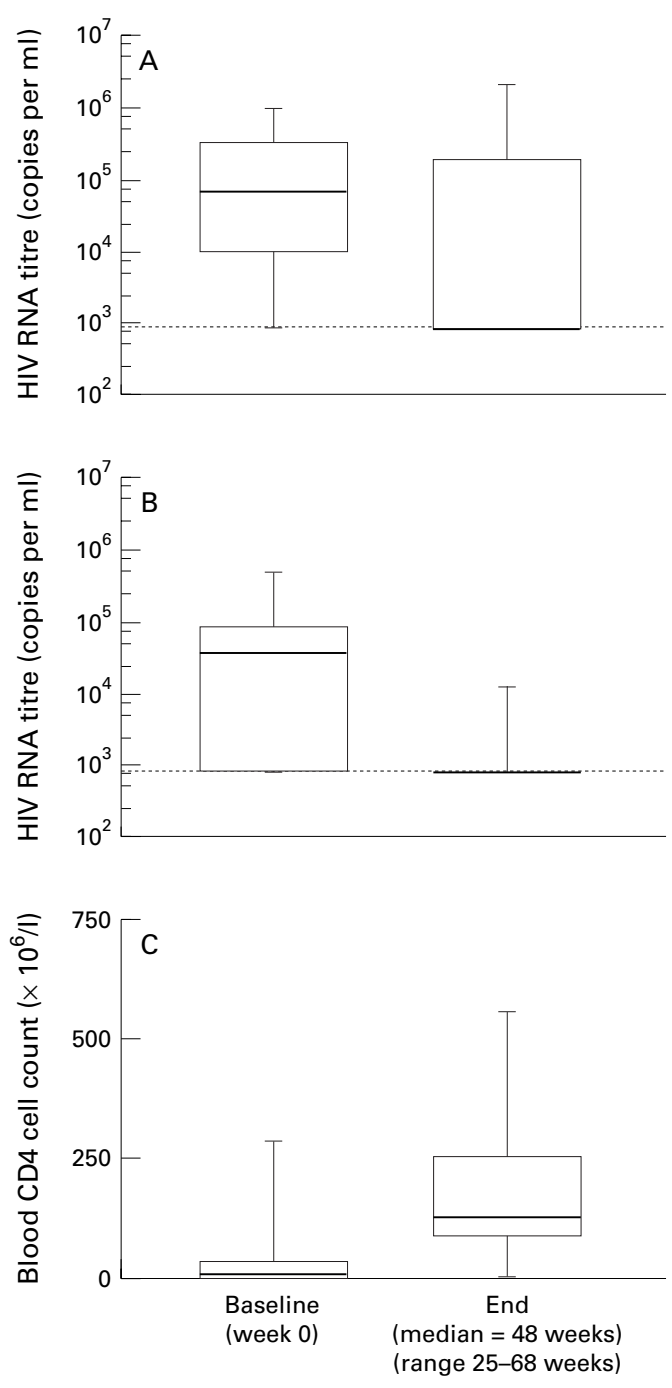

Figure 1 Distribution of blood $(A)$ and seminal plasma (B) HIV-1 RNA titres and blood CD 4 cell counts (C) in 12 nucleoside inhibitor experienced men before (baseline) and following (end) indinavir combination therapy. Data are median and interquartile range; bars represent the maximum and minimum values. Broken line, lower limit of detection at 800 copies/ml. (Values less than the assay cut off were assigned values of 799 copies $/ \mathrm{ml}$.)

the simian immunodeficiency virus ${ }^{7}$ suggest that the inoculum viral titre is an important determinant of transmission efficacy. Therefore, the potential benefit of reducing genital tract viral loads, and consequently diminishing transmission risk, via antiretroviral therapy, is immense. In two previous studies ${ }^{45}$ the viral loads in seminal fluid and blood from the majority of patients were reduced to below the baseline within a few weeks of initiation of therapy and this reduction was maintained during the study periods of 15-25 weeks. Our study involved follow up of patients for up to 68 weeks of treatment, and six of the 12 patients had evidence of viral persistence or resurgence by the end of the study period. Analysis of longitudinal samples (not shown) demonstrated that in one patient virus resurgence was detected in the seminal fluid tract before the blood plasma. In this patient viral breakthrough may have arisen because of the production of resistant viruses within the genital tract with subsequent dissemination to the peripheral circulation. We are unable to ascertain definitively whether apparent failure of 
indinavir to reduce and maintain HIV RNA below the detectable level was associated with the presence of drug resistant viruses, reduced drug uptake within the genital tract, or through patient non-compliance, although results of patient questioning did not support the latter reason. Administration of zidovudine has been shown to produce equivalent reductions of blood and seminal fluid HIV-1 RNA titres, and this would be consistent with the finding that inhibitory concentrations of zidovudine are achieved within semen following oral delivery. ${ }^{8}$ We have recently shown that seminal plasma concentrations of the protease inhibitors saquinavir and ritonavir are less than $5 \%$ of the corresponding blood plasma concentration and are variably above the in vitro $\mathrm{IC}_{90},{ }^{9}$ although similar data for indinavir are not available. This disparity may encourage the differential emergence of protease inhibitor resistant virus. Future sequence analysis of the populations present in each sample should clarify this. Outgrowth of protease inhibitor resistant viruses in NRTI experienced individuals has been reported previously.

Our study concentrated on the effects of treatment upon viral RNA loads. However, the potential role of cell associated virus in sexual transmission cannot be overlooked. ${ }^{7}$ Significant levels of cell associated HIV-1 provirus are detectable in seminal fluid samples ${ }^{10}$ and, more importantly, there is some evidence to show that semen from HIV RNA negative individuals who have undergone potent combination therapy can still harbour detectable proviral DNA. ${ }^{11} 12$ Therefore, determination of both viral RNA and proviral DNA titres should be included in future studies aimed at determining the effects of antiretroviral therapy within the genital tract.

In conclusion this study shows that elimination of blood and genital tract HIV RNA, using combination therapy in individuals who have previously received extensive antiretroviral therapy, is frequently unachievable and, therefore, these individuals could represent a serious transmission source of drug resistant viruses. It is important to underline the potential risk of sexual transmission of HIV despite undetectable viral load results. This work was supported in part by grant number Council, United Kingdom.

Contributors: D Pillay and J K Ball conceived the study, superContributors: D Pillay and J K Ball conceived the study, super-
vised the laboratory work, carried out the data analysis, and were involved in writing the paper; R Curran and T Rowe carried out the laboratory work; G Sontag, $M$ Youle, and G Moyle contributed to the study design and coordinated data collection at the study centres; finally, G M Beards and W L Irving contributed to the writing of the paper.

1 Zhu T, Wang N, Carr A, et al. Genetic characterisation of human immunodeficiency virus type 1 in blood and genital secretions: evidence for viral compartmentalisation and selection during sexual transmission. F Virol 1996;70:3098107

2 Lee T-H, Sakahara N, Fiebig E, et al. Correlation of HIV-1 RNA levels in plasma and heterosexual transmission of HIV-1 from infected transfusion recipients. $\mathcal{F}$ AIDS Res Human Retroviruses 1996;12:12100-4.

3 Eron JJ, Vernazza PL, Johnston DM, et al. Resistance of HIV-1 to antiretroviral agents in blood and seminal plasma: implications for transmission. AIDS 1998;12:F181-9.

4 Gupta P, Mellors J, Kingsley L, et al. High viral load in semen of human immunodeficiency virus type 1-infected semen of human in men at all stages of disease and its reduction by therapy with protease and non-nucleoside

5 Vernazza PL, Gilliam BL, Flepp M, et al. Effect of antiviral treatment on the shedding of HIV-1 in semen. AIDS 1997; 11:1249-54.

6 Van Gemen B, Kievits T, Schukkink R, et al. Quantification of HIV-1 RNA in plasma using NASBA during HIV-1 primary infection. F Virol Methods 1993;43:177-88.

7 Joag SV, Adany I, Li Z, et al. Animal model of mucosally transmitted human immunodeficiency virus type 1 disease: intravaginal and oral deposition of simian/human immunodeficiency virus in macaques results in systemic infection, elimination of $\mathrm{CD}^{+} \mathrm{T}$ cells and AIDS. F Virol 1997;71: 4016-23.

8 Henry K, Chinnock BJ, Quinn RP, et al. Concurrent zidovudine levels in semen and serum determined by radioimmunoassay in patients with AIDS or AIDS-related complex. $¥ A M A$ 1988;259:3023-6.

9 Taylor S, Back DJ, Workman J, et al. Poor penetration of the male genital tract by HIV-1 protease inhibitors. AIDS (in press)

10 Xu C, Politch JA, Tucker L, et al. Factors associated with increased levels of human immunodeficiency virus type DNA in semen. $\mathcal{F}$ Infect Dis 1997;176:941-7.

11 Coombs RW, Speck CE, Hughes JP, et al. Association between culturable human immunodeficiency virus type 1 (HIV- 1) in semen and HIV-1 RNA levels in semen and blood: evidence for compartmentalization of HIV-1 between semen and blood. F Infect Dis 1998;177:320-30.

12 Zhang $\mathrm{H}$, Dornadula G, Beumont $\mathrm{M}$, et al. Human immunodeficiency virus type 1 in the semen of men receiving highly active antiretroviral therapy. N Engl f Med 1998; 339:1803-9. 